\title{
LA AGUDEZA HAYDNIANA \\ ENEL PRINCIPIO DRAMÁTICO DE LOS \\ CUARTETOS OP. 59 DE BEETHOVEN
}

\author{
Mónica Gudemos \\ Universidad Nacional de Córdoba - Argentina \\ Email:mgudemos@gmail.com
}

\section{Resumen:}

En este trabajo se observa la asimilación dramática que Beethoven, principalmente en sus cuartetos de cuerda de estilo compositivo medio, hizo de la agudeza (Witz) haydniana. Agudeza que, para la crítica de finales del siglo XVIII significó fuente de vituperación ya sea por la falta de rigor técnico o por la falta de "decoro», permitió establecer discursivamente ciertos niveles de sentido que más tarde Beethoven profundizaría en la definición de su propio estilo.

\section{Palavras-clave:}

Agudeza haydniana. Retórica beethoveniana.

Haydn's wit in the drammatic principle of Beethoven's op. 59 Quartets

\section{Abstract:}

In this work we observe the dramatic assimilation that Beethoven, mainly in his quartets of string of medium compositional style, made of the Haydnian sharpness (Witz / Wit). Sharpness that, for the critique of the last decades of the 18th century meant a source of vituperation either due to the lack of technical rigor or the lack of "decorum", allowed to establish discursively certain levels of meaning that later Beethoven would deepen in the definition of his own style.

\section{Keywords:}

Haydnian wit. Beethovenian rhetoric. 
1. Año de produccióny primera publicación, respectivamente.

2. Rasumowsky. En la bibliografía especializada aparecen ambas formas de escribir el apellido del conde.

3. Los cuartetos Op. 33 fueron compuestos por Haydn en ocasión de la visita a Viena del Gran Duque Pablo de Rusia y su esposa María Feodorowna, princesa de Württemberg. Los cuartetos Op. 59, por su parte, fueron dedicados por Beethoven al Conde Razumovsky [Andrej Kyrillovič Razumovskij], embajador ruso en la corte de Viena. Sabido era entonces que Razumovsky profesaba una particular admiración por Haydn, principalmente por sus cuartetos de cuerdas, de los que llegó a interpretar partes de segundo violín. Promovió las presentaciones del Cuarteto Schuppanzigh, el más importante de Viena en aquel tiempo, contribuyendo incluso hacia 1808 con su mecenazgo.

4. Véase al respecto Dahlhaus 1982, 1989; Krebs 1998.

5. "Aus jedem Motiv des ersten Themas lassen sich Fermente gewinnen, die sich innerhalb des gross angelegten und interessanten harmonischen Bauplans zur Ausarbeitung eignen. Thematische Modelle knüpfen in der Exposition ein Netz von wechselseitig aufeinander bezogenen $\mathrm{El}$ ementen"(LOCKWOOD, 2012, p. 250).
Más allá de las relaciones socio-históricas que podemos establecer entre los cuartetos de cuerdas Op. 33, conocidos como los cuartetos GliScherzi (1781/1782)릴 de Joseph Haydn y los cuartetos de cuerdas Op. 59 Razumovsky ${ }^{2}$ (1806/1808) de Ludwig van Beethoven ${ }^{3}$, estética y estilísticamente observamos que ambas obras, cada una en su tiempo, fueron compuestas "con arreglo a principios totalmente nuevos", utilizando una afirmación del mismo Haydn con respecto a sus seis cuartetos «rusos» (Rosen 1986: 28). Principios que, no obstantelos años transcurridos entre la producción de ambas obras, responden a una particular concepción dramática del ritmo y una interesante configuración de las estructuras morfológicas que les permitió generar diferentes niveles de relatividad formal. Todo esto obró en función de una paulatina revisión conceptual del modus operandi "arquitectónico", según Dahlhaus ${ }^{4}$, de las articulaciones micro- y macro-formales (heredero aún de ciertos preceptos barrocos, como observa Ludwig Nohlen 1881)en pro de una lógica del discurso, cuyo Grundgedanke [idea fundamental] confirió a las relaciones intrínsecas no sólo una naturaleza procesual dramática nunca antes explorada técnicamente en música instrumental, sino particularmente una organicidad de sentido gestáltico que requirió nuevas formulaciones armónicas. Tales formulaciones posibilitaron superar la linealidad regular de los arcos sintácticos de la funcionalidad, permitiendo así mayor independencia y ponderación significativa de los materiales en cada uno de sus elementos constitutivos. Haydn y Beethoven supieron aprovechar técnicamente muy bien estas particularidades, transformándolas en uno de los recursos compositivos más sobresaliente de sus estilos: la generación de tramas discursivas retroalimentadas por la dramaticidad propia de los materiales temáticos, que Ernst T. A. Hoffmann (analizando la quinta sinfonía de Beethoven) describió como ese «innere tiefe Zusammenhang" [profunda conexión interior] otorgante desentido (HOFFMANN, 1963: 34-51).

Sin duda, los cuartetos Op. 33 de Haydn fueron en esto verdaderos precursores. En efecto, la observación queLockwood (2012: 252) $)^{5}$ hace con respecto ala red de elementos mutuamente relacionados por patrones temáticos, motívicamente retroalimentados y sustentados por un interesante plan constructivo armónicamente diseńado, del primero de los 
Op. 59 de Beethoven, bien se aplicatambién a los Op. 33 de Haydn.

En ellos puede constatarse una profunda transformación temática a través de procesos muy efectivos, desde la variación encadenada a partir de la fragmentación motívica hasta el desfasamiento métrico y la complejización textural por un hábil entramado contrapuntístico que mantiene la unidad discursiva, al tiempo que permite una interesante exploración tonal mediante un ritmo armónico de mayor dinamismo.

Con respecto a esto último, analizando la producción de Haydn inmediata anterior y contemporánea a los cuartetos GliScherzi, es claro observar la asimilación que el compositor hizo de los recursos compositivos de sus óperas y Singspiele en la música instrumental de cámara ${ }^{6}$, específicamente en lo que se refiere al sentido dramático que los materiales cobran en el discurso cuando se trabaja, sin prejuicios formales, a partir de sus fuerzas inherentes. Así lo comprendieron Haydn y Beethoven. Técnicamente, esto fue posible a partir de una operatividad motívica más dinámica e integrada con los diferentes parámetros compositivos y una «economía de medios» que magistralmente promovió Haydn en sus cuartetos a partir de su Op. 33 y, principalmente, su Op. 50 compuesto para el Rey de Prusia Frederick William IIen 1787.

Beethoven logró una buena asimilación de ambas técnicas, particularmente en su segundo periodo estilístico, siguiendo prácticas haydnianas con respecto a las estrategias contrapuntísticas de amplio espectro ${ }^{7}$, que llegó a desarrollar y canalizar con particular habilidad en sus cuartetos Op. 59, hasta lograr una máxima abstracción en su último periodo estilístico en obras como el Cuarteto en Do\#m Op. 131.

En este trabajo me propongo demostrar cómo la agudeza haydniana, técnica y retóricamente comprendida a partir de los procedimientos compositivos, fue dramáticamente asimilada por Beethoven en su periodo medio, principalmente en sus composiciones para cuarteto de cuerdas. Esa agudeza, que para la crítica setentista del siglo XVIII significó fuente de vituperación ya sea por la falta de rigor técnico o por la falta de «decoro» (LUCAS, 2008, p.136137), permitió establecer discursivamente ciertos niveles de sentido que más tarde Beethoven profundizaría en la definición de su propio estilo. Para ello y por la extensión
6. Es interesante, no obstante, constatar que la crítica contemporánea no observaba estas asimilaciones técnicas, sino la falta de lo que podríamos denominar «el decoro» con respecto al efecto que la obra debía tener sobre la audiencia. Por ejemplo, Johann (Critischer Entwurf einer auserlesenen Bibliothek für den Liebhaber der Philosophie und schönen Wissenschaften, 4th ed. (Berlin, 1771) señaló que incluso un "conocedor medio" podía detectar la "extraña mezcla delo cómico y lo serio" que predomina "en la música de Haydn, los hermanos Toëschi, Cannabich,Filz, Pugnani y Campioni”. Los críticos de Haydn, no se oponían a los elementos cómicos per se, sino más bien a lamezcla ostensiblemente inapropiada que el compositor hacía deelementos serios y cómicos dentro de una misma obra (BONDS, 1991, p. 83).

7. Pienso aquí, particularmente, en la integración haydniana de procedimientos fugados en secciones de desarrollo, dotándolos de carácter dramático. Beethoven asimila esta práctica sin restringirla sólo a las secciones de desarrollo y la potencia a través de innovadores planos tonales, como aquellos que promoverían lo que Schönberg (analizando el comienzo del último movimiento del Op. $59 \mathrm{~N}^{\circ}$ 2) llamó die ebendeTonalität [tonalidad suspendida] (Harmonielehre, Wien 1911). 
8. Término que utilizo aquí para referirme a las particularidades sintácticas que definen las operatividades compositivas básicas de un determinado discurso musical.

9. La amistad y colaboración profesional entre Romberg y Beethoven fue registrada en varias fuentes desde los tiempos en que ambos formaban parte de la orquesta de la corte del Electorado en Bonn. Véase Solomon 1998. exigida para este trabajo, me centraré en ejemplos puntuales que me servirán como referencia ilustrativa.

\section{LA AGUDEZA HAYDNIANA EN LA IMPRONTA BEETHOVENIANA}

El planteo macro- y micro- formal del Allegretto vivace e semprescherzando del primero de los tres cuartetos Op. 59 nos permite observar claramente la original agude$z a$ haydniana en la concepción dramática beethoveniana. Micro-formalmente, la "conducta» ${ }^{8}$ de los materiales que configuran la articulación fraseológica básica de este movimiento (compases 1 a 8) constituye el núcleo primario del impulso procesual del principio de desarrollo, que anima una estructura de sonata caracterizada por su gran extensión y fuerza dinámica (LOCKWOOS, 2012, p. 251).Ya en su tiempo, el afamado cellista y viejo conocido de Beethoven, Bernhard Romberg (1767-1841), consideró tal conducta «ein rotes Tuch» [paño rojo, como el utilizado para azuzar los toros] acicateando desde el principio del movimiento el desenvolvimiento de su envergadura (MOSER, 1955, p. 1064; LOCKWOOS, 2012, p. 251). Una envergadura que habría que esperar hasta la composición del Scherzo de la 9na. Sinfonía (1818-1824), situado también como segundo movimiento, para establecer comparaciones operativas.

Pocos en el auditórium e incluso pocos intérpretes contemporáneos a los Op. 59 así lo entendieron, aun el mismo Romberg 9 , quien habría presuntamente "pataleado" (trampelte) ante una parte instrumental tan "inconsistente» (LOCKWOOD, 2012, p. 248).

Si bien esta "conducta» se origina en el comienzo mismo del cuarteto, la relación de oposición discursiva entre estatismo y «vuelo gestual» que se establece entre el cello y el 2 do. violín, respectivamente, genera en este scherzo un antecedente de dramático dinamismo. Este principio dramático está esencialmente sustentado en la agudeza de Haydn puesta de manifiesto en su ya mencionado Op. 50. Bien se sabe que Frederick William II era un buen aficionado a la música instrumental, aunque su desempeño como cellista no fuese «destacable». Lo interesante es que el primero de los cuartetos Op. 
50 a él dedicados, en su primer movimiento, comienza con un solo para cello de una nota repetida para que el soberano pudiese «lucirse» sin temor al fracaso. Ahora bien, este solo instrumental que habría sido decepcionante para el cellista de la corte de los Esterházy, Joseph Franz Weigl(1740-1820), en mi opinión constituye una exquisita "agudeza» haydniana (que de modo magistral se explota compositivamente en los retruécanos de generación dramática de la exposición) al servicio de un «decoro» formal y discursivo importante.

En efecto, la configuración dramática que Haydn logra en esta estructura de sonata con el estatismo del ostinato del cello y el vuelo sintáctico de los materiales en los otros instrumentos se mantiene, como en el ejemplo beethoveniano, a lo largo de todo el movimiento en un entramado temático de gran amplitud. Podríamos bien referirnos aquí al característico desarrollo temático haydniano o Durchführung propio de finales del siglo XVIII pleno de Humor $^{10}$, en el que la variación y la fragmentación del material, su secuenciación y la economía de medios, así como el ingenioso juego de las disonancias (que en este cuarteto no alcanzan una funcionalidad estructural como en el primer movimiento del Op. 33 $\mathrm{N}^{\circ} 1$ en Sim) son las principales operatividades compositivas.

Beethoven, por su parte, en el Scherzo que estudiamos del Op. $59 \mathrm{~N}^{\circ} 1$, apuesta a sostener un discurso permanentemente renovado hasta los límites de la propia «identidad material» de sus componentes estructurales. El trabajo textural tensionando los preceptos del contrapunto clásico, los planos armónicos (aproximándose en este caso al concepto de tono modalidad ampliada, sin abandonar por cierto la articulación clásica), el estupendo trabajo sobre la percepción métrica (enmascarándola con un ingenioso juego de intensidades, véase por ej. compases 333 a 350), la concepción estructural de los adornos y su competencia discursiva mediante el principio de variación al que somete el ritmo temático, así como el desarrollo permanente de las inercias inherentes de los materiales son los artificios constructivos beethovenianos.
10. Pienso aquí en el sentido en el que lo analiza Bonds (1991,p. 63) a partir del concepto de «aniquilación» propuesto por Jean Paul Richter (1763-1823): "So spricht z.B. Sterne mehrmals lang und erwagend fiber gewisse Begebenheiten, bis er endlich entscheidet: es sei ohnehin kein Wort davon wahr. Etwas der Keckheit des vernichtenden Humors Ahnliches, gleichsam einen Ausdruck der Welt-Verachtung kann man bei mancher Musik, z.B. der Haydnschen, vernehmen, welche ganze Tonreihen durch eine fremde vernichtet und zwischen Pianissimo und Fortissimo, Presto und Andante wechselnd stürmt." Jean Paul Richter 1963, vol. 5) “Jean Paul's concept of 'annihilation' can be understood on several levels. What is 'annihilated' most immediately is that which precedes an abrupt change. Within the dynamics of the temporal arts, however, it is also the reader's or listener's anticipation of what is to follow that is annihilated. The very fact that an idea is discussed 'at length and weightily leads us to expect that it will be of some import in the larger scheme of things. In the case of instrumental music, we expect the flow of a work to follow certain conventions of tonality, dynamics, and tempo. In thwarting these expectations, Haydn does not "disdain' his listeners in the sense of ignoring them -indeed, he calculates and plays upon their anticipations masterfully- but the effect of such devices, as Jean Paul observes, is to create a sense of separateness and distance from the world at large, including the listener" 
11. Particularmente, parto del "Prinzip der entwickelnden Variation, das der thematisch-motivischenStrukturzugrundeliegt" que trata Carl Dahlhaus en Schönberg. Variationen für Orchester (1963).

12. Aquí no dedico espacio para el tratamiento del magistral trabajo rítmico que hace Beethoven en este movimiento en función de su carácter scherzante. Sugiero consultar Headlam1985.

13. En Inglaterra, alrededor de 1810 , Radicati le comenta a Thomas Appleby con respecto a los cuartetos Op. 59: "Haben sie diese hier auch bekommen? Beethoven, wie die Welt sagt und ich glaube, ist musikvoll; das hier ist keine Musik. Er zeigte sie mir im Manuskript, und auf seine Bitte schrieb ich einige Fingersätze hinein, daß er sicherlich diese Werke nicht für Musik ansehen könne, worauf er mir antwortete: 'O, sie sind auch nicht für Sie, sondern für eine spätere Zeit!"' (TDR II, S. 536f, apud LOCKWOOD, 2012, p. 244 y $417 / 15$, p. 1).

14. "Desenvolver estruturas individuais dentro de moldes escritos".
Beethoven integrará tales artificios en un pensamiento continuo de carácter teleológico, sustentando la estructura micro- y macro- formal a través del principio de variación en desarrollo ${ }^{11}$, profundizando el sentido dramático con el que Haydn concibió sus materiales. Un sentido dramático que en las obras del Kappelmeister de los Esterházy jamás llegó a transformar la materialidad de su discurso al punto de establecer nuevas naturalezas temáticas, que exigieran la derivación espacio-temporal de los puntos de tensión hasta el final mismo de la obra para su resolución, como sí lo hizo Beethoven sin importarle, por cierto, el «decoro dieciochesco».

Lo que resulta interesante es cómo Beethoven, pese a los profundos cambios que opera en este movimiento estructurado a partir del principio de oposición de sonata o de disonancia estructural, mantiene el sentido scherzante a través de una forzada articulación regular que, poco a poco, va cediendo ante el avance dramático de las fuerzas inherentes de los materiales, de las precipitadas "caídas» que desestructuran los procesos de densificación textural (por ej. compases 110 a 114), de las sorpresivas suspensiones dramáticas en pausa al mejor estilo haydniano tras alcanzar un punto de clímax creciente (por ej. compases 170y 238), de las abruptas yuxtaposiciones entre los homofónicos batidos rítmicos y las cantilenas texturadas (por ej. compases 296 a 304), de las fugaces animaciones fugadas y la permanente presencia del batido de semicorcheas, que sólo la maestría de Beethoven mantuvo al límite mismo de la saturación ${ }^{12}$.

Un original juego al que, aún sin comprenderlo, el violinista Felix Radicati habría contribuido con determinadas digitaciones y con una crítica que dio origen a la célebre respuesta del maestro de Bonn: "O, siesindauchnichtfürSie, sondernfüreinespätereZeit!” [Oh, ellas tampoco son para usted, sino para un tiempo posterior]. ${ }^{13}$

\section{EL PERSPICAZ «DECORO»BEETHOVENIANO}

Después de las búsquedas estéticas y estilísticas del movimiento arriba tratado, encontrarse con un Menuetto Grazioso en el tercer movimiento del cuarteto Op. $59 \mathrm{~N}^{\circ} 3$ en DoMllama mucho mi atención. Como en los Scherzi del Op. 33 de Haydn, este movimiento presenta la tendencia de "de- 
sarrollar estructuras individuales dentro de moldes escritos" (Lucas 2008: 157) ${ }^{14}$. Si bien su articulación temática primaria sigue los preceptos estructurales del Menuetto de finales del dieciocho $^{15}$, la continuidad del discurso dada por la constante precipitación de una variación rítmica, métrica y motívica da cuenta de la capitalización de las operatividades compositivas alcanzadas por Beethoven en los scherzos de los cuartetos Op. $59 \mathrm{~N}^{\circ} 1$ y 2 y de la Eroica, entre otras obras, principalmente del principio de variación en desarrollo. Principio que superó en el planteo formal y conceptual de este movimiento el estatismo procesual del intercambio de voces y la «elongación suspendida" del Fortspinnung barroco.

Por cierto, la concepción compositiva de este Menuetto dista no sólo técnicamente, sino también retóricamente de aquel que Beethoven incluyera en la primera de sus tres sonatas Op. 2, publicadas en Viena en 1796 y que dedicara a Haydn. Beethoven habría comenzado a trabajar en la impresionante sonata en Fam en 1793 (LOCKWOOD, 2012, p. 70; KRAMER, 1980). No dudo que el estilo beethoveniano se perfilaba ya claramente en esta sonata a través de una particular elección de los recursos compositivos en el marco del género. Pero entre tales recursos, tampoco dudo que la búsqueda "efectista" a través de la modulación inesperada, el silencio de carácter dramático, así como el gusto por transgredir la regularidad estructural de la fraseología clásica son asimilaciones de la herencia haydniana (ROSEN, 1986, p. 129). Ahora bien, el concepto de "efecto" en el Beethoven del Menuetto del Op. 59 trasciende yael concepto de lo experiencial sensible (Empfindung) de aquellas inadecuaciones «indecorosas» alto-cómicas o puntuales witzige Einfälle o incluso de lo que yo llamaría aquellas eventuales geistreiche Einfälle (ocurrencias) para el deleite de los oídos «agudos» que aún nos es posible observar en el Menuetto del Op. $2^{16}$. El «efecto» en Beethoven asumiría ya en la primera década del XIX un carácter de distanciamiento reflexivo, de complejización discursiva permanentemente acicateada por un principio dialéctico que aspiraba (sehnen) no a la satisfacción (Wohlgefallen) de lo agradable (angenehm), sino a la satisfacción por la sensación (Sehnsucht) de la totalidad (Vollendung) a través del hecho inteligible, aunque sea el más mínimo de esa totalidad. No desconozco en esto el acercamiento conceptual que Beethoven tuvo respecto
15. Koch, 2001[1802], IV, 2, 18, 5 (citado también por LUCAS, 2008, p. 157 ss.).

16. Por ejemplo, la exquisita expectativa dramática que genera al final dela parte B del Menuetto, después de haber recurrido al gesto cadencial primario; el engrosamiento textural por cuartas en acordes en primera inversión del trío y el cambio agógico de los acordes en blancas con punto potenciando el color disonante de un cromatismo que parece perderse en las profundidades de la percepción; el cambio de planos armónicos y el interesante juego dinámico que confunde la audición con fugaces enmascaramientos métricos, poco «decorosos» para un Menuetto.

17. Particularmente a su Critica del Juicio. Aunque se estima que Beethoven sólo accedió superficialmente a la filosofía kantiana a través de fórmulas simplificadas popularizadas (SOLOMON, 1998, p. 53), creo que su relación con dicha filosofía pudo ser más estrecha y reflexiva. Sus permanentes contactos con los francmasones de la logia Illuminati en Bonn lo acercaron ya a la obra de Kant. Por las prohibiciones vigentes, los integrantes de esta logia se convocaron desde 1785 en la Lese und Erholungs-Gesellschaft (Sociedad de lectura y recreación). La Cantata para la muerte del emperador Joseph II fue compuesta por Beethoven en 1790 por un pedido expreso de esta so- 
ciedad. Entre los integrantes de la Gesellschaftestaban sus amigos Nikolaus Simrock, Franz Ries, vonSchall, Johann Peter Eichoff,Johann Joseph Eichoff, incluso también su maestroNeefe, "quien era uno de los jefes del grupo (su Lokaloberer) y que publicó su propio semanario desde abril de 1784"(SOLOMON,1998, p.35). Posiblemente la matriculación de Beethoven en 1789 junto a Reicha y Kügelgenen la Universidad de Leipzig tuvo que ver con sus veladas en la taberna Zehrgarten. A dicha taberna se anexaba la famosa librería de la viuda Anna María Koch(SOLOMON, 1998, p. 52), en la que se reunían profesores universitarios, diferentes intelectuales y radicales de todas las clases y edades a tratar temas entre los que el pensamiento kantiano no estaba excluido. No olvidemos que en esa universidad realizó sus estudios de derecho el mismo Neefe, quien pudo también instarlo a instruirse en temáticas legales y filosóficas. Aunque extrañamente Beethoven no quiso asistir a las conferencias que sobre Kant se dieron en Viena en 1790, ni siquiera a instancias de su amigo Wegeler, la ávida lectura que el compositor hizo de obras sobre filosofía $\mathrm{y}$ arte (en especial ópera) ha sido ampliamente documentada.Véase, también, Konrad 2015.

18. En particular de su exitoso libro Herzensergiessungen eines kunstliebenden Klosterbruders [1796] y de su obra póstuma Phantasien über die Kunst [1799]. Ambas obras, en las que colaboró activamente Tieck, ejercieron de la filosofía kantiana (KANT, 1990) ${ }^{17}$ y del pensamiento de Wilhelm Heinrich Wackenroder (1773-1798) ${ }^{18}$ y de su colaborador Ludwig Tieck (1773-1853).

Lo que resulta interesante es que el discurso beethoveniano, en cierto modo y en su forma de concebir la esencia de los materiales en su «innere tiefe Zusammenhang» otorgante de sentido, actualiza la impronta de la agudeza haydniana, no a partir del concepto de humor al que Schillingen 1835 se refiere en su Encyclopädie ( Laune»" ${ }^{19}$, que implica lo caprichoso y antojadizo), sino a partir del concepto propiamente scherzante del «Witz», pero en el sentido del término inglés «Wit» [ingenio] tal como lo expone Alfons Klüpfel (2004: 17) ${ }^{20}$, esto es, en un sentido que vincula conceptualmente el ingenio con el talento y el buen juicio de entendimiento. Un sentido que en Beethoven adquiere la perspicacia del agudo juego que implica el término «Schärfe» [agudeza], involucrando significativamente tanto la inteligible nitidez del gesto retórico y la sutileza del detalle que nos sorprende, infringiendo más allá de las formas el «decoro» dieciochesco del Menuetto, hasta la perspicacia de un planteo intelectual que ya no apela a lo risible, sino a la incómoda insatisfacción de lo inasible.

La materialidad del Menuetto del Op. $59 \mathrm{~N}^{\circ} 3$ está impregnada de la materialidad de los movimientos anteriores, incluso en lo más sutil de las falsas relaciones que promueve la diastematía de la segunda sección del Menuetto. Si bien comprendo este movimiento en el marco de la lógica general del cuarteto, continuando el tempo instalado por el Andante y estableciendo una coda puente hacia el poderoso cuarto movimiento, un Allegro molto que conceptualmente implica el cierre no sólo de este cuarteto sino de toda la serie Op. 59; llama poderosamente mi atención la elección por parte de Beethoven de un «gesto galante» para este movimiento. Pienso que precisamente en esa elección radica la «invitación al juego». Ahora bien, las reglas han cambiado.

Como observa Webster (1980, p. 127), este Menuetto habría sido originalmente pensado en FaM, no en DoM, y su trío en RebM. Pienso que su diseño definitivo respondióal sentido global del ciclo y a un particular criterio compositivo que,en este cuarteto, debía contemplar la importante relatividad formal con la que opera Beethoven desde el comienzo. Relatividad que, en el primer movimiento, incluye una Intro- 
duzione generativa de carácter temático con indefinición tonal hasta que el cello busca inteligibilidad armónica en el grave, equilibrándose texturalmente con el primer violín. Además, le sigue una ágil segunda introducción temática que se inserta con un solo de violín en el comienzo mismo del Allegro vivace, marcando las pautas rítmicas esenciales y acrecentando el arco de tensión mediante una secuenciación suspendida/ interrumpida por remanentes materiales de la Introduzione, interpolados de tal modo que generarán un sustrato esencial permanente en todo el cuarteto. No olvidemos, por cierto, la proyección de los arcos tonales que articularán y, al mismo tiempo, sostendrán como arbotantes la estructura general de la obra.

Ahora bien, esta relatividad formal que sin duda tomó desprevenidos hasta los más formados instrumentistas y oyentes de las primeras décadas del siglo XIX (incluso, como vimos,al mismo Radicati), me recuerda en cierto modo al efecto que causó en los oyentes de la década del ' 70 del siglo XVIII el final del último movimiento del Op. $33 \mathrm{~N}^{\circ} 2$ de Haydn. Esa agudeza haydniana para movilizar al oyente más preparado (BONDS, 1991, p. 70) está también presente en los Op. 59 de Beethoven, aunque el sentido dramático que la anima dista ya considerablemente.

La proyección de los arcos tonales que arriba menciono, genera al escuchar el Andantecon moto quasi Allegretto en Lam del Op. $59 \mathrm{~N}^{\circ} 3$ la sensación de un cierto «exotismo» ${ }^{21}$, que se potencia por la tensión discursiva que emerge de la coexistencia de un fluido entramado contrapuntístico con esquemas iterados y puntos de pedal. Este Andante exige no sólo una atenta audición, sino también el ejercicio de una introspección de análisis retrospectivo para hacerse inteligible en el sentido general del cuarteto. Todo capitalizándose conceptual y técnicamente en un pensamiento compositivo que sufrirá una fuerte y "graziosa» inflexión al aparecer un Menuetto que producirá una nueva tensión discursiva (no sólo armónica, por cierto) y una magistral precipitación hacia el Allegro molto final.

Lo observado hasta ahora forma parte de ese juego global, de esa agudeza beethoveniana (Schärfe) en la que el Menuettodel Op. $59 \mathrm{~N}^{\circ} 3$, con su gracia diociochesca, se ha transformado discursivamente en el punto quasi simétrico de inflexión una fuerte influencia en el cambio de siglo. En 1994 fueron editadas por Reclam de Stuttgart.

19. E1 término Laune, en Altdeutsch (alemán antiguo), tiene un sentido sensiblemente diferente al que Schilling registra en su Enciclopedia de 1835. En el siglo XVIII, según Johann Georg Sulzer en la Allgemeine TheoriederschönenKünste (1776), define Laune como: Un temperamento en el que un sentimiento vagamente agradable o desagradable es tan predominante que todas las ideas y expresiones del alma están impregnadas por ella" (BONDS, 1991, p. 61).De allí era la creencia de que la disposición de un artista estaría indirectamente, pero inevitablemente presente en su obra.

20. "Im englischen «wit» finden wir es noch im Sinne von Verstand, Weisheit, Schlagfertigkeit oder «Mutterwitz», im Sinne des französischen «esprit»" (KLÜPFEL, 2004, p. 17).

21. Término propuesto por Lockwood(2012, p.253). 
estructural, formando parte con el Allegro molto final de una unidad muy alejada conceptualmente de la función que competía a los scherzos y minuetos.

En un análisis «de superficie» nos encontramos en este tercer movimiento con un planteamiento formal clasicista, con estructuras repetidas y una vuelta $D a$ Capo tradicional, pero con un lenguaje novedoso que inserta el principio de desarrollo en el B del Menuetto y que exige un virtuosismo particular en el Trío al que, a su vez, da una longitud poco «decorosa». A esto se suma una Coda asumida no como un ornato de cadenzas, sino como la continuación del proceso de desarrollo iniciado en el B del Menuetto, instando a una resolución armónica que la precipita al vigoroso final. Las regiones de inestabilidad armónica, así como la función estructural que le compete en el cuarteto, le confieren al Trioese ethos excêntrico que Lucas (2008, p. 158-159) observa en el Scherzo del Op. $33 \mathrm{~N}^{\circ} 4$ de Haydn, pero potenciado por la lógica de continuidad dramática arriba explicada que, sin conciencia de ella, este Menuetto sería, en sí mismo, inasible en su propuesta compositiva. Ese «potencial inasible» constituye la agudeza beethoveniana.

Tal vez sea preciso aquí modificar la anterior observación sobre la presencia en este movimiento de la tendencia haydniana de "desarrollar estructuras individuales dentro de moldes escritos", puesto que estaríamos frente a la «Schärfe» beethoveniana de jugar con la tensión generada entre la constricción de un molde y la presión ejercida sobre dicho molde por un lenguaje dramático que lo desborda peligrosamente. Una tensión que sólo puede decantar en la violenta dinámica del último movimiento. Lo hace de forma evidente para generar el efecto crítico sobre las antiguas formas, utilizando una de las estructuras más tradicionales, incluso "fuera de moda» ya a finales del siglo XVIII. El decoro de las formas ya no interesa a Beethoven en este estadio compositivo, tampoco hablaría aquí de la búsqueda de determinadas incongruencias sutiles para el oído instruido y atento como gesto de Humor o Alto-cómico, sino de agudeza en el sentido dramático beethoveniano que, no obstante tener sus antecedentes en la agudeza haydniana, exige ahora reflexión. 


\section{EPÍLOGO I: LA AGUDEZA DEL ECLECTICISMO BEETHOVENIANO}

Permítaseme aquí, antes de concluir este trabajo, hacer una necesaria digresión y apartarme de los cuartetos Op. 59. Por lo general, para hablar de las influencias recibidas por Beethoven durante sus años de formación, solemos partir de dos instancias que la historia refiere al respecto, ambas tomadas, respectivamente, de las manos de Christian Gottlob Neefe (1748-1798), organista de la corte del Elector de Colonia en Bonn desde 1781 y maestro de Beethoven posiblemente desde ese mismo año (MOSER, 1955, p. 857), y de Joseph Haydn.

Se dice que de las manos de Neefe tomó Beethoven el espíritu de Bach ("Bachs Geistaus Neefes Händen") 22 y de las de Haydn el de Mozart ("Mozart's Geistaus Haydens Händen") $)^{23}$.

Pero sabemos que recibió también otros "espíritus" antes de dejar definitivamente Bonn. El del mismo Haydn de manos de Neefe, quien se declaró su más ferviente admirador cuando el Kapellmeister de los Esterházyse detuvo en Bonn en su paso hacia Londres en 1790, así como el de los más relevantes compositores del momento. No olvidemos, por otra parte, que nada influye más en la primera formación que el propio entorno y en esto acuerdo con Konrad (2015) cuando observa que Beethoven no esperó a radicarse en Viena para desarrollarse como artista ${ }^{24}$. Ya en Bonn se desempeñó como compositor, músico de corte y activo crítico de su entorno sociocultural. Un desempeño que fue posible gracias a su ávida voluntad de aprender de todo aquello que lo rodeaba y de cuanta partitura cayese en sus manos. Tampoco olvidemos que su interés compositivo no se centró exclusivamente en la música instrumental. La música vocal, particularmente la ópera, llamaba poderosamente su atención. Los escenarios de Bonn en las décadas de los '80 y los '90 del siglo XVIII "prestaron tablas» a las óperas de Gluck, Cimarosa, Mozart, Sacchini, Paisiello, Benda, Salieri, Grétry, Pergolesi, Gossec, Dalayrac, Dittersdorf, Holzbauer... y, por supuesto, de Neefe. El eclecticismo estilístico que Maynard Solomon (1998) observa en los primeros bocetos y trabajos completos de Beethoven, se debió sin duda a la puesta en práctica por parte del joven estudiante de los recursos compositivos analizados
22. Lockwood 2012, p. 23. No dudo que Neefe aplicó sus recursos pedagógicos siguiendo el estudio del Clavecín bien temperado de J. S. Bach, tal como él mismo menciona en la comunicación que dirigiera a la Magazin der Musik el 2 de marzo de 1783, propiedad del “Kieler Gluckanhängers und Klopstockverehrers Prof. Karl Friedrich Cramer" (MOSER, 1955, p. 227). En dicha comunicación Neefe presentaba por primera vez a su alumno, argumentando que, si continuaba como comenzó, se convertiría en el segundo Mozart. Magazin der Musik [Revista de

Música] fue una publicación periódica que apareció entre 1783 y 1789. Lo que dudo es que Beethoven (posiblemente de 12 años, aunque en la comunicación se mencione de 11 años; véase Solomon 1989, 34-35) haya sido debidamente instruido en las técnicas del contrapunto. En efecto, en dicha comunicación Neefe sólo dice que "toca muy hábilmente el clavecín y lo hace con fuerza, lee muy bien a primera vista $y$-para decirlo de una vez- ejecuta principalmente El clavecín bien temperado de Sebastian Bach", no especifica que conociera sus procedimientos compositivos y experimentara a partir de ellos. Es más, al llegar a Viena en 1792 Beethoven llevaba consigo serias dificultades técnicas en escritura musical con las que el maestro Haydn, poco sistemático para la enseñanza, no le pudo ayudar. Fueron Johann Schenk (1753-1836) en 1793 y, principalmente, Johann Albrechtsberger en 1794 quienes introdujeron sistemáticamente a 
Beethoven en el estudio del contrapunto, no sin dificultad debido a la natural predisposición improvisatoria y autosuficiencia creativa (Salieri y el mismo Albrechtsberger mencionaban también la «obstinación») del estudiante (véase Lockwood 2015, Solomon 1998 y Nottebohm 1971). Por otra parte es necesario recordar que cuando Mozart o Haydn, entre otros compositores notables de la época, hablaban de Bach, se referían principalmente a C.P.E. Bach (cuya obra tenía mayor presencia social que la de su padre en la segunda mitad del S. XVIII). Ambos respetaban su libro de enseñanza Ensayo sobre el verdadero arte de tocar el teclado (1753), que posiblemente Beethoven conoció. Lo cierto es que buena parte del «espíritu de Bach» recibido por Beethoven habría sido el de Carl Philipp Emanuel. Véase Fundación de la red "C.P.E. Bach 1714" http:// www.cpebach.de (30-012017).

23. Tal como refiere la nota que el joven Conde Waldstein escribió en 1792 en el álbum de recuerdos de Beethoven al momento de su segunda partida a Viena.

24. "Dass der „Bonner“ Beethoven keine Larve war, aus der erst in Wien der wirkliche Künstler geschlüpft ist, diese Behauptung sollte nach unseren Bemerkungen weniger als bilderstürmende These denn vielmehr als berechtigte Annahme für die Biographie wie für den schöpferischen Werdegang des Komponisten unstrittig sein" (KONRAD, 2015) de la producción contemporánea. No obstante y aún siendo evidente en las obras de Bonn el sistema de "composiciónensayo" que Beethoven ponía en práctica como un modo de asimilación de los recursos formales estudiados, principalmente en su música instrumental, y de los recursos expresivos y dramáticos en su música vocal, se observa en ellas el perfil de determinados recursos idiomáticos propios, que se verán claramente reflejados en obras posteriore ${ }^{25}$. Por ejemplo, las influencias de Mozart y C.P.E. Bach, entre otros,en su música instrumental son notables, tal como puede observarse en los Cuartetos para piano y cuerdas de 1785 (WoO 36) con respecto a la fuerte impronta estilística mozartiana (por la que tal vez nunca fueron publicados). No obstante, la originalidad melódica beethoveniana que se perfila en estas obras será aludida en obras posteriores como las sonatas del Op. 2, Op. 13 y Op. 27, por ejemplo. Pero, si dela agudeza en el eclecticismo beethoveniano hablamos, tal vez uno de los casos más interesantes para estudiar sea la Cantata Joseph (WoO 87), como se la denomina comúnmente.

Tal como menciona Solomon, en esta cantata la influencia de Christoph Willibald Gluck (1714-1787) es evidente en la concepción tímbrica en general y técnico-instrumental en la forma de escribir para cuerdas y vientos en particular. Esto no es menor cuando dicha concepción trata de sustentar una retórica elegíaca. El joven Beethoven trabajó para ello sobre las obras de un compositor esencialmente dramático como Gluck, para el que en sus aproximadamente 107 óperas «jeder Ton nur dem Drama diene» (MOSER, 1955, p 427) ${ }^{26}$. El timbre fue fundamental en la definición estilística de Beethoven para la conducción inteligible de las líneas discursivas en el entramado textural. Tanto, que incluso en sus sonatas para piano ${ }^{27}$ logró un amplio espectro de densidades sonoras y matices, que explotaron hábilmente las posibilidades técnicas de generación de armónicos del instrumento (la sonata Pathétique Op. 13, publicada en 1799 y dedicada al príncipe von Lichnowsky, es un buen ejemplo de lo dicho).

La concepción melódica mozartiana en las arias para soprano y bajo es igualmente relevante; pero el Pathos que les da sentido es propiamente beethoveniano y aún cuando algunos reconozcan las apothéoses de la escuela de Rameau y la impronta mannheimniana de Holzbauer en los unísonos introductorios a las arias (SOLOMON, 1998, p. 65; SCHIEDERMAIR, 1925), la pro- 
fundidad dramática con la que Beethoven los asimila compositivamente es completamente nueva. En la forma en que Beethoven logra ese eclecticismo asimilador de los diferentes «modos de hacer» a los que tiene acceso en su momento-lugar histórico, de comprender culturalmente la producción de sus contemporáneos y de aquellos maestros que consideró referentes en su formación es ya una «agudeza estilística» de fuerte impacto para el propio entorno en el que se desarrollaba.

Por ello, para quienes a un consideran que el "Beethoven de Bonn", el compositor de esta Cantata, nos es "nuestro Beethoven" (KONRAD, 2015), aquel que conocemos y pre-conceptualizamos en nuestros análisis, por la sencilla razón que su estilo no «responde» a los preceptos clásicos a él adjudicados y sus aspiraciones no se «co-responden» con las expectativas estéticas definidas por una posteridad «clasificada» como romántica por la literatura de finales del siglo XIX y principios del siglo XX; e incluso para aquellos contemporáneos al Beethoven de $1813^{28}$ y para quienes hacia la década de los '60 del siglo XIX aun observaban subjetivamente el eclecticismo de sus obras del periodo de Bonn con cierto aire academicista descalificador, adjudicándolas a un Beethoven que todavía no es "el nuestro": "Eristnochnichtunser Beethoven, aberderwerdendekündigtsichan" (Alexander Wheelok Thayers: Beethoven-Biographie [1866], apud KONRAD, 2015); para ellos parece ir dirigida la nota que Johannes Brahms envió en Mayo de 1884 al crítico Eduard Hanslick (1825-1904). Tal nota fue redactada después de conocer e inspeccionar el manuscrito de la cantata, nuevamente descubierto en una subasta, reconociendo "de punta a punta» el estilo beethoveniano: „Ständeaberkein Nameaufdem Titel, mankönnteaufkeinen Andernrathen- es ist Alles unddurchaus Beethoven! Das schöneedle Pathos, das Großartige in Empfindungund Phantasie, das Gewaltige, auchwol Gewaltsameim Ausdruck, dazu die Stimmführung, die Declamationund in beidenletzterenalle Besonderheiten, die wirbeiseinenspäteren Werkenbetrachtenundbedenkenmögen” (KONRAD, 2015). Donde se veía inconsistencia técnica y estilística, Brahms reconoció la «Schärfe» beethoveniana, jugando con la tensión generada entre la constricción de un molde y la presión ejercida sobre dicho molde por un lenguaje dramático que lo desborda peligrosamente, como antes dije.
25. Para este punto consultar Solomon 1998, Lockwood 2012, Webster 1980.

26. "Cada sonido sirve sólo al drama”.

27. Sabemos que Beethoven, como IgnazMoscheles (1794-1870), Johann NepomukHummel (1778-1837), Ferdinand Ries (1784-1838) y John Field (1782-1837), entre otros, exigió un particular dinamismo mecánico en los pianos para lograr un control de los matices como jamás lo hubiesen requerido Mozart o Haydn; tal vez de allí su permanente interés por los cambios técnicos de este instrumento, prefiriendo en varias ocasiones los pianos ingleses por su «presión al tacto» y su potencia.

28. Año en que el compositor Johann NepomukHummel adquirió el manuscrito de la Cantata en una subasta. El manuscrito permaneció oculto por muchos años, hasta que apareció en otra subasta en 1884, año de su primera interpretación. $\mathrm{Al}$ año siguiente fue estrenada en Bonn. 
29. Epigramático: E1 Soneto, fragmento (GOETHE [tomo 1], 1991, p. 1105).

30. Epigramático: Condición Fundamental (GOETHE [tomo 1], 1991, p. 1188).

\section{EPÍLOGO II: EL SONETO DE GOETHE}

Hacia 1800 Goethe componía uno de sus sonetos ${ }^{29}$, ofreciéndolo a las generaciones del nuevo siglo como un legado de su experiencia y maestría:

Del arte practicar los modos nuevos, sagrado deber es que se te impone; según el ritmo y el compás prescritos, moverte tú también como yo puedes.

Seguramente Beethoven habría observado en él una profunda contradicción: ¿Por qué debería moverse según el ritmo y el compás prescritos si quería del arte los modos nuevos practicar?; tal vez le hubiera llamado a reflexión la posibilidad de que, aún utilizando los modos preexistentes, podría él plantear algo nuevo y propio.Si bien como músico de oficio sabía que si de naturaleza y de arte hablaba como condición fundamental ${ }^{30}$ debía siempre tener delante de sus ojos la obra de quienes como él buscaron su propio lenguaje, sabía también que no podía moverse como ellos si quería decir algo distinto.Abrevó en cuanta fuente fue necesario para situarse en un tiempo en el que debía capitalizar los recursos adquiridos, pero sólo en la medida en que éstos le ayudaron a concebir su particular y nuevo modo de hacer.

\section{AGRADECIMIENTO}

Mi agradecimiento a la Dra. Mónica Lucas por compartir con gran generosidad su conocimiento.

\section{REFERENCIAS BIBLIOGRÁFICAS:}

BONDS, Mark Evan. Haydn, Laurence Sterne, and the Origins of Musical Irony. In:Journal of the American Musicological Society, v. 44, n.1, 1991, p. 57-91. Disponible en:http://www. jstor.org/stable/831728. Consulta: 4 feb. 2017.

DAHLHAUS, Carl. Schönberg. VariationenfürOrchester. Meisterwerke der Musik. München: Wilhelm Fink Verlag, 1963. 
University Press, 1982.

Esthetics of music. Cambridge: Cambridge Nineteenth-century music. Berkeley: University of California Press, 1989.

GOETHE, Johann Wolfgang. Obras completas. México: Edición Aguilar, 1991.

HEADLAM, Dave. A Rhythmic Study of the Exposition in the Second Movement of Beethoven's Quartet Op. 59, No. 1. In: Music Theory Spectrum, v. 7,1985, p. 114-138.

KANT, Immanuel. Critica del Juicio. Madrid: Espasa-Calpe, 1990.

KLÜPFEL, Alfons. Witz und Humor in der Musik. In: AfSMagazin 18,2004, p. 16-18.

KOCH, Johann Christoph. MusikalischesLexikon. Kassel [Frankfurt]: Bärenreiter [August Hermann der Jüngere], 2001 [1802].

KONRAD,Ulrich.Der'Bonner' Beethoven. Vortrag 2609-2015. InstitutfürMusikforschung der Universität Würzburg. Disponible en: https://www.youtube.com/ watch?v=I0Rvd0BZYyM. Consulta: 30 ene. 2017.

KRAMER, Richard. Das Organische der Fuge: On the Autograph of Beethoven's String Quartet in F Major, Op. $59 \mathrm{~N}^{\circ}$ 1. In: WOLFF, Christoph (ed.). The String Quartets of Haydn, Mozart, and Beethoven. Studies of the Autograph Manuscripts. Cambridge: Mass,: Harvard University Press, 1980, p. 223265.

KREBS, Wolfgang. ZumVerhältnis von musikalischer Syntax und Höhepunktsgestaltung in der zweitenHälfte des 19. Jahrhunderts. In: Musiktheorie 1998, p. 31-41. Disponible en: http://www.wk-wkw.de/texte/aufs/A-Syntax-Hoehepunktsgestaltung-19Jh.pdf. Consulta: 10 ene. 2017. 
LOCKWOOD, Lewis. Beethoven.Seine Musik. SeinLeben. Kassel: Bärenreiter/Metzler, 2012.

LUCAS, Mónica. Humor e Agudeza em Joseph Haydn quartetos de cordasop. 33. São Pablo: Annablume, Fapesp, 2008.

MOSER, Hans Joachim. MusikLexikon. Bände I-II. Hamburg: Musikverlag Hans Sokorski, 1955.

NOHL, Ludwig. Allgemeine Musikgeschichte. Leipzig: Druck und Verlag von Philipp Reclam, 1881.

NOTTEBOM, Gustav. Beethoven's Unterrichtbei J. Haydn, Albrechtsberger und Salieri: nach den Original-Manuskripten (Beethoven's Studien, Bd.1). Wiesbaden [Leipzig]: Sändig [Rieter-Biedermann], 1971 [1873].

RICHTER, Jean Paul. Vorschule der Ästhetik. Werke, Series I, vol. 5. München: Carl Hanser, 1963.

ROSEN, Charles. El estilo clásico. Haydn, Mozart y Beethoven. Madrid: Alianza Música, 1986.

SCHIEDERMAIR, Ludwig. Der Junge Beethoven. Leipzig, 1925.

SOLOMON, Maynard. Beethoven. New York: Schirmer Trade Books, 1998.

WEBSTER, James. Traditional Elements in Beethoven's Middle-Period String Quartets. In:Beethoven, Performers, and Critics. Detroit: Wayne State University Press, 1980, p. 94-133. 